\title{
DOES BALANCE IMPROVE AFTER THE APPLICATION OF TAI CHI EXERCISE PROGRAM IN POST- MENOPAUSAL WOMEN WITH OSTEOPOROSIS?
}

\author{
Ema Lašinytė, Vilma Mauricienė, Alfonsas Vainoras, Kristina Berškienė \\ Lithuanian University of Health Sciences, Institute of Sports, Kaunas, Lithuania
}

\begin{abstract}
Background. Osteoporosis is an emerging medical and socioeconomic threat characterized by a systemic impairment of bone mass, strength, and microarchitecture. Due to degenerative changes in morphology and structure, the amount of bone fragility and fractures increases affecting balance function. Tai Chi is a traditional Chinese mindbody exercise that has the potential to overcome the impairments associated with frailty.

Methods. The study involved 13 post-menopausal women with osteoporosis. All subjects participated in Tai Chi exercise program which lasted for eight weeks. Two, one-hour group training sessions were taught per week. Balance assessment was performed three times: before Tai Chi exercise program, after four weeks and after eight weeks of the application of Tai Chi exercise program. Balance measurements were conducted using platform "Sigma Balance Pad" and BESS test.

Results. Research findings of "Sigma Balance Pa" showed statistically significant changes $(p<.05)$ in eyes opened condition while in eyes closed condition no significant changes were observed $(p \geq .05)$. Results of BESS test revealed statistically significant improvement of static balance after Tai Chi exercise program $(p=.033)$.

Conclusions. Research results demonstrated balance improvement: body sway and center of pressure displacement speed decreased after Tai Chi exercise program. Further future research of reliability and validity of the BESS in subjects with osteoporosis should be considered.
\end{abstract}

Keywords: static balance, Sigma Balance Pad, Tai Chi Wudang.

\section{INTRODUCTION}

$\mathrm{O}$ steoporosis is a common disease characterized by a systemic impairment of bone mass, strength, and microarchitecture (Zhou et al., 2014). It is an emerging medical and socioeconomic threat resulting in increased propensity of bone fragility and fractures (Curtis, Moon, Dennison, Harvey, \& Cooper, 2015).

Postural control, described as the inherent capacity to maintain the center of mass within the base of support, which defines the stability limits (the edges of the operational areas through which the center of mass can be displaced without the need to move the base of support) (Brech, de Foncesa, Bagnoli, Baracat, \& D'Andrea Greve, 2013), is critical to the maintenance of balance and avoidance of falls. When properly functioning, this multi-level neural control system produces stable balance and gait (Wayne et al., 2014). However, vertebral fractures cause a change in body posture due to increased kyphosis of the thoracic spine, which causes a forward displacement of the center of mass of the trunk (Smulders, van Lankveld, Laan, Duysens, \& Weerdesteyn, 2011) such that it approximates the stability limits (Brech, de Foncesa et al., 2013). As a result, a constricted limits of stability boundary or uncontrolled center of gravity movement can impair functional balance and constrain safe performance of daily activities such as reaching, bending, walking, gait initiation, and moving from sit-to-stand (Li, 2014). 
Tai Chi is a multi-component mind-body exercise that is grounded in the holistic model of traditional Chinese medicine and integrates balance, flexibility, and neuromuscular coordination training with a number of cognitive components such as deep abdominal breathing, relaxation, focused body awareness, imagery and multi-tasking (Wayne et al., 2014). Tai Chi exercises are composed of a series of graceful motions linked together in a continuous sequence so that the body is continuously shifting from one foot to another. According to Li (2014), Tai Chi is capable to expand the limits of stability boundaries. This is important for improving balance and reducing the risk of falling among older people, especially for those with osteoporosis ( $\mathrm{Li}, 2014)$. Despite the fact that many studies presented positive effects of Tai Chi on body balance, there is still a lack of papers which include computer-based balance analysis as well as there is no research which includes BESS test to measure the balance changes after Tai Chi exercise program.

The aim of this study was to evaluate balance changes in post-menopausal women with osteoporosis after Tai Chi exercise program.

\section{METHODS}

Subjects. Post-menopausal women $(n=13)$ with osteoporosis were included in the study. The average age was 74.2 years (the range of the age was 67-81 years), the average height was $158.6 \mathrm{~cm}$, the average weight was $67.1 \mathrm{~kg}$. Subjects belonged to Kaunas district osteoporosis club "Uola". Participation in the study was voluntary. The study received the approval of the Kaunas Ethics Center of Lithuanian University of Health Sciences (No. BEC - SR(M) - 134). All participants signed a statement of informed consent after receiving information about the course of investigation and its methods.

Research protocol. The static balance of the subjects was assessed using BESS balance test and objective indicators of body balance were conducted using platform "Sigma Balance Pad". Balance assessment was performed three times: before Tai Chi exercise program, after four weeks and after eight weeks of application of Tai Chi exercise program.

"Sigma Balance Pad". This platform is a device for balance and proprioception examination and training. The device assesses any change in the position of the platform, processes these changes and transmits data wirelessly in real time to a computer with the software.

Assessment of balance included two conditions: standing on both legs on the plate for $60 \mathrm{~s}$ with eyes opened and for $30 \mathrm{~s}$ with eyes closed. Patient's safety was ensured during the whole procedure. Changes in center of pressure (COP) displacement were registered based on six objective indicators:

- in lateral direction - average deviation $\mathrm{X}$ (cm),

- in anterior-posterior direction - average deviation Y $(\mathrm{cm})$,

- COP displacement speed in lateral direction - average velocity $\mathrm{X}(\mathrm{cm} / \mathrm{s})$,

- COP displacement speed in anteriorposterior direction - average velocity $\mathrm{Y}$ $(\mathrm{cm} / \mathrm{s})$,

- path length $(\mathrm{cm})$,

- area $\left(\mathrm{cm}^{2}\right)($ Rutkauskiene, Piščalkienè, Gintilienè, Zachovajevienè, \& Kavaliauskienè, 2012).

BESS balance test. The BESS has criterionrelated validity with force plate measures. Reliability of the total BESS score and individual stances ranges from poor to moderate to good, depending on the type of reliability assessed. It is known that the BESS is valid to detect balance deficits where large differences exist (Bell, Guskiewicz, Clark, \& Padua, 2011). Even we have not found any articles where BESS test would be used for measuring balance changes after Tai Chi exercise program, we decided to include this test in the study protocol to find out whether BESS test results can be sufficiently informative to help understand and draw conclusions about actual balance changes after exercise program.

BESS balance test consists of three standing position and two different surfaces on which subject stands. Testing must be conducted without shoes. Standing positions:

- double leg stance: the feet are flat on the testing surface approximately pelvic width apart.

- single leg stance: subject stands on the nondominant leg with the contralateral limb held in approximately $20^{\circ}$ of hip flexion, $45^{\circ}$ of knee flexion and neutral position in the frontal plane.

- tandem stance: one foot is placed in front of the other with heel of the anterior foot touching the toe of the posterior foot. 
The subject's non-dominant leg is in the posterior position. Leg dominance should be determined by the person's kicking preference.

The two different surfaces include both a firm (ground) and foam (Airex pad) surface.

The leg dominance was determined before testing by asking a control question "If you had to kick the ball, which leg would you do this?" The subject's preferenced leg in the answer was considered to be dominant. The investigation for failing to respond to this question, or in case of doubt on the accuracy of the response, the participant was actually asked to kick the ball.

The balance in three standing positions was tested first on firm, and then - on foam surface. Subject's stance should consist of the hands on the iliac crests, eyes closed and a consistent foot position depending on the stance. Testing in each standing positions lasted for $20 \mathrm{~s}$ and the number of errors (deviations) from the proper stance were calculated during that time. Counting errors was begun only after the subject had assumed the proper testing position. The errors were considered: moving the hands off the hips, opening the eyes, step, stumble or fall, abduction or flexion of the hip beyond $30^{\circ}$, lifting the forefoot or heel off of the testing surface, remaining out of proper testing position for more than $5 \mathrm{~s}$. The maximum total number of errors for any single condition was 10 . If a subject committed multiple errors simultaneously, only one error was recorded. After testing each position, the number of errors was marked in the test protocol. Errors made on firm and foam surface (separately for each surface) as well as total BESS score (errors on firm + foam surfaces) were calculated (Bell et al., 2011).
Tai Chi exercise program. The exercise program lasted for eight weeks. Sessions were held twice a week (16 Tai Chi sessions in total). The duration of each session was $60 \mathrm{~min}$. Tai Chi sessions were initiated with 5-10 min duration of introductory warm-up exercises, 40-45 min of the main part of Tai Chi and 5-10 min of relaxation exercises. The main part of Tai Chi session consisted of eight basic Tai Chi movements as well as static stances for the body's center of mass training. Moreover, dynamic exercises, integrating coordinated feet - hand movements and breathing, were taught. Dynamic exercises were selected from Wudang Tai Chi Sanfeng $13^{\text {th }}$ form, according to functional capacity of participants. Exercises got progressively more difficult by the course.

Statistical analysis. Statistical analysis was conducted using SPSS 22.0. The non-parametric Friedman's test for K-related samples was used. The level of statistical significance was set at $p<.05$.

\section{RESULTS}

The findings of the "Sigma Balance Pad" showed that statistically significantly changes were found only in opened eyes condition. The deviation in $\mathrm{X}$ axis results were better after eight weeks of Tai Chi exercise program $\left(\chi^{2}=1.091\right.$ (2), $p=.032$ ), while the deviation in $\mathrm{Y}$ axis did not change significantly $\left(\chi^{2}=2.476(2), p=.29\right)$, which means that body sway after Tai Chi program in lateral direction was less compared to pre-exercise program results.

After eight weeks of the program, results in velocity in $\mathrm{X}$ axis were statistically significantly

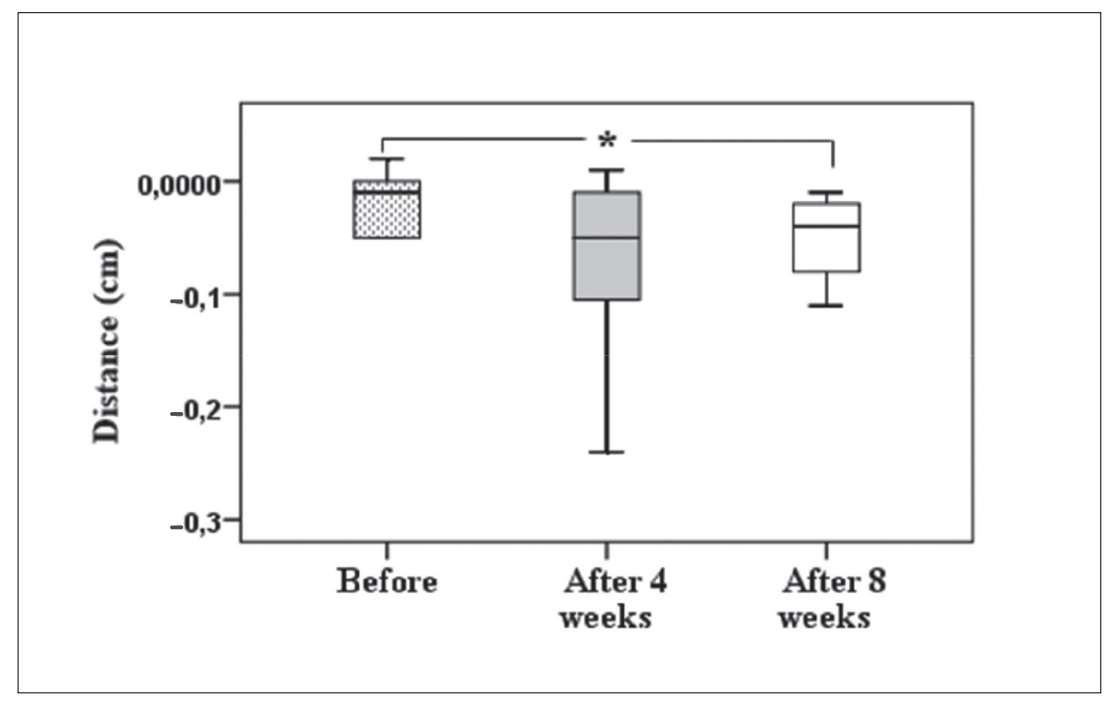

Figure 1. Changes in deviation in $\mathbf{X}$ axis before and after Tai Chi exercise program 
lower compared to pre-program testing $\left(\chi^{2}=1.227\right.$ (2), $p=.012)$ as well as significant changes were measured in comparison to the results after four and eight weeks of Tai Chi exercise program $\left(\chi^{2}=\right.$ $1.091(2), p=.032$ ).

Changes in velocity in $\mathrm{Y}$ axis were also found. Statistically significantly lower results were observed after eight weeks of the program $\left(\chi^{2}=\right.$ $1.227(2), p=.012$ ).

As velocity in $\mathrm{X}$ and $\mathrm{Y}$ axes represents the COP displacement speed, the lower speed means that person is more stable on the platform during the test (which is related to better balance).

After the assessment of balance, we found that results of path length and area improved after Tai Chi program: the path length statistically significantly decreased $\left(\chi^{2}=1.182(2), p=.017\right)$ after eight weeks of exercise program and the area was also smaller after eight weeks compared to primary testing and this change was statistically significant $\left(\chi^{2}=1.182(2), p=.017\right)$. It is known that the smaller the path length and area are, the better the balance is.

As mentioned earlier, no statistically significant changes were found in balance assessment in eyes closed condition $(p \geq .05)$.

Comparing the results between opened and closed eyes conditions, the results in eyes closed condition were worse than in eyes opened condition both before and after Tai Chi exercise program.

The results of BESS test revealed no statistically significant changes in firm surface
Figure 2. Changes in velocity in $X$ and $Y$ axes before and after Tai Chi exercise program

Note. $* p<.05$

Figure 3. Changes in path length before and after Tai Chi exercise program

Note. $* p<.05$
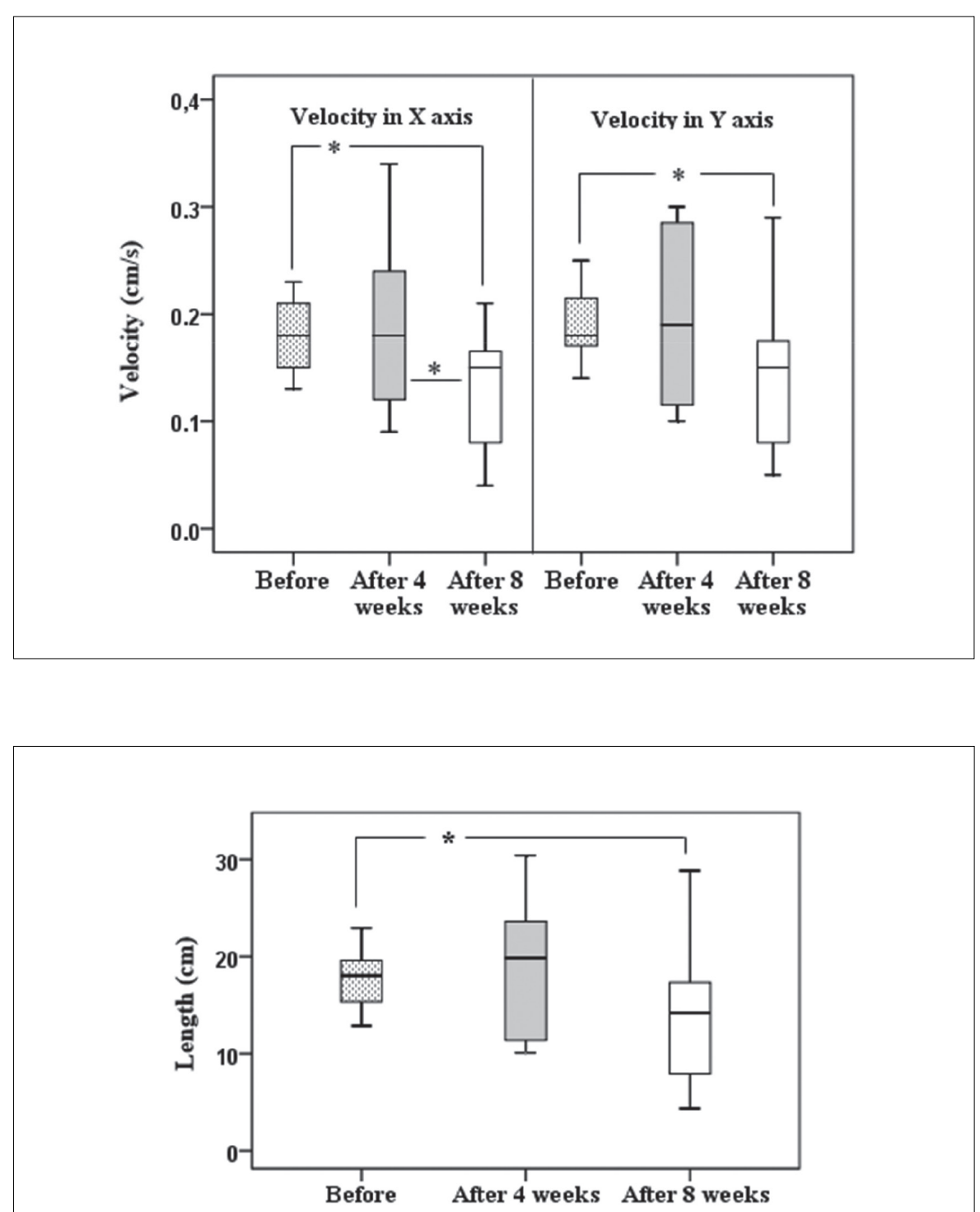


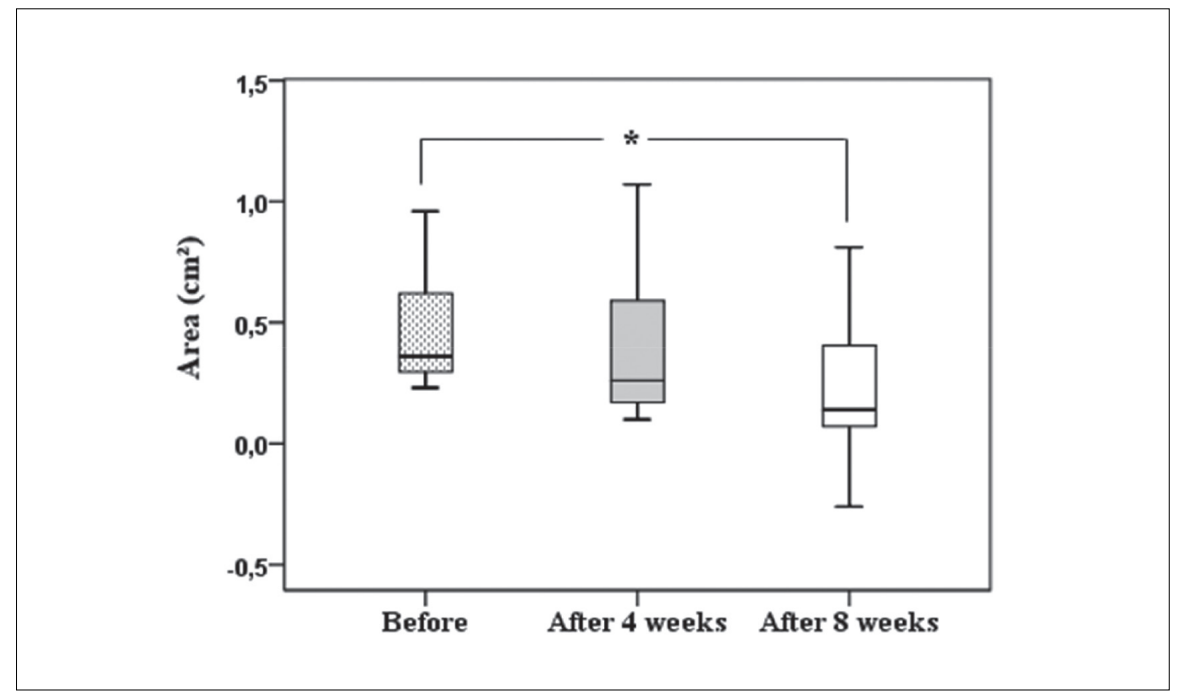

Figure 4. Changes in area before and after Tai Chi exercise program

Note. ${ }^{*} p<.05$.

test condition after Tai Chi exercise program ( $p \geq .05$ ). Significantly lower number of errors was made in double leg stance position on foam surface $(p=.012)$ after eight weeks of Tai Chi program. A tendency of lower numbers of errors made in tandem stance on foam surface was observed, although no statistically significant changes were found ( $p \geq .05$ ). Furthermore, total number of errors on foam surface statistically significantly decreased $(p=.027)$ as well as decreased total number of errors (firm + foam surface) $(p=.033)$ after eight weeks of Tai chi exercise program. All results of the test are presented in the Table.

Table. Results of BESS test

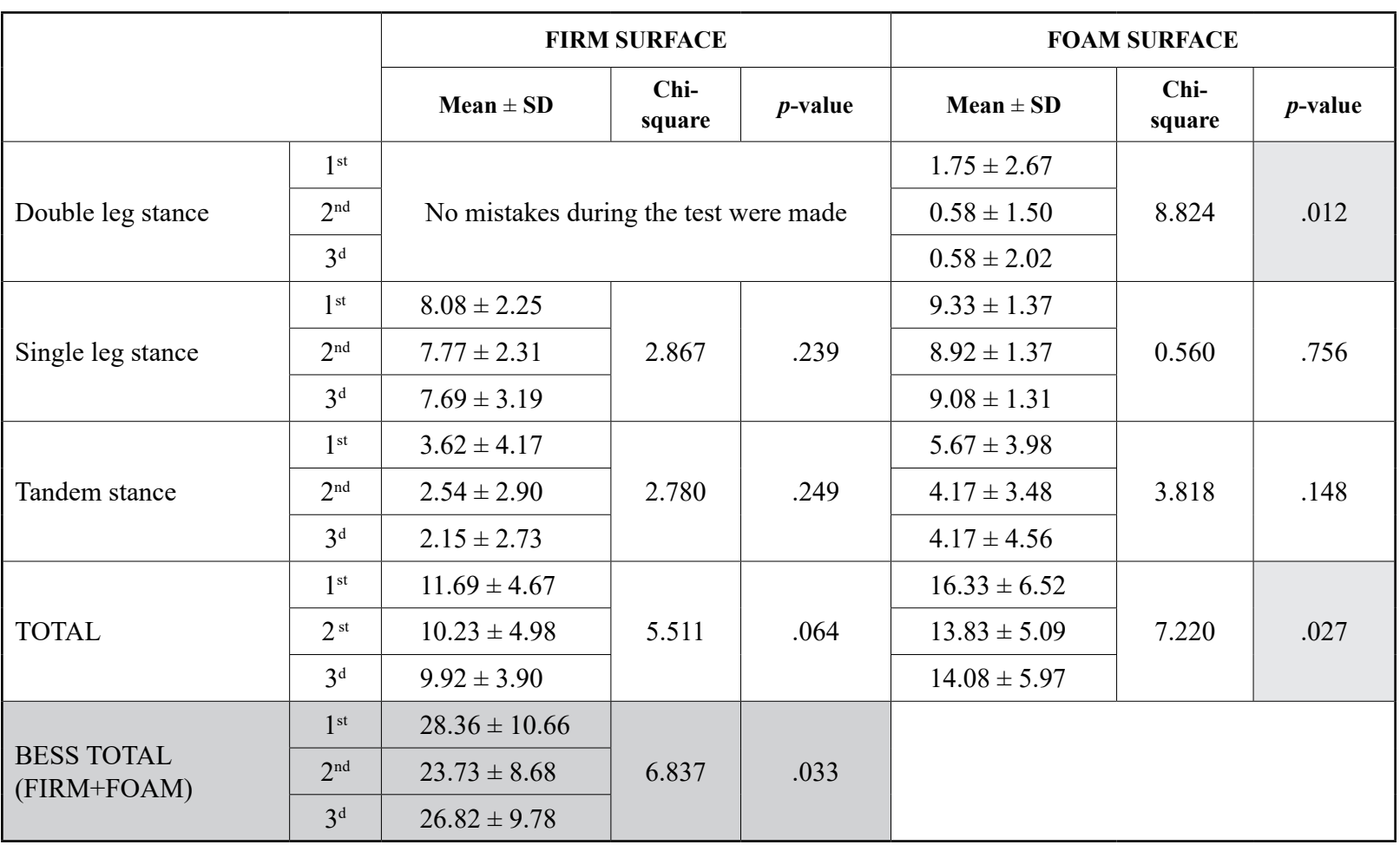

Note. $1^{\text {st }}$ - before Tai Chi program;

$2^{\text {nd }}-$ after four weeks of Tai Chi program;

$3^{\mathrm{d}}-$ after eight weeks of Tai Chi program. 


\section{DISCUSSION}

In their study, Lelard et al. (2010) compared the respective effects of Tai Chi exercise program and a balance training program on static postural control and walking ability. Static postural control was assessed via the measurement of the center of pressure sway in eyes open and eyes closed conditions. The results of the study showed that performance in the eyes closed condition was lower than in the eyes open condition in pretest and posttest for the balance training and Tai Chi groups. The results of this study are similar as in our study no statistically significant changes were observed in the assessment of balance in the eyes closed condition. In another study (Wayne et al., 2012), the impact of Tai Chi exercise on multiple fracturerelated risk factors in post-menopausal osteopenic women were investigated as participants were assigned to nine months of Tai Chi training. Quiet standing fall-predictive sway parameters were assessed in the study. Changes in sway parameters were observed: average sway velocity $(p=.027)$ and anterior-posterior sway range $(p=.014)$ were significantly improved by Tai Chi training (Wayne et al., 2012). Our study results showed statistically significantly improved parameters in the eyes opened condition: deviation in $\mathrm{X}$ axis $(p=.032)$ after eight weeks of Tai Chi exercise program, average velocity in $\mathrm{X}$ axis after four $(p=.012)$ and eight weeks $(p=.032)$ of Tai Chi and velocity in $\mathrm{Y}$ axis $(p=.012)$ after eight weeks of Tai Chi exercise program. As mentioned earlier, no statistically significant changes were observed in eyes closed conditions. As it is known, tests with eyes closed are known to be more "provocative" than tests performed with eyes open (Wayne et al., 2012). Therefore, it can be assumed that the two-month Tai Chi exercise program is insufficient for positive changes in sway parameters to be observed testing balance in eyes closed condition.
Results of BESS test total scores showed statistically significant improvement of static balance after Tai Chi exercise program $(p=.033)$ while no significant changes were observed in foam surface test results. Although results of double leg stance on a foam surface improved statistically significantly after exercise program $(p=.012)$, however, it was a reason why we also received a significant improvement in total scores section of foam surface $(p=.027)$. As mentioned earlier, we could not find other studies which used BESS test for measuring balance changes after Tai Chi exercise program, so our research results cannot be compared with other researchers' results.

Analyzing BESS test's pros and cons in our study protocol, it can be assumed that this test includes quite complicated testing positions and conditions which might have been too difficult for our participants.

Furthermore, sophisticated Tai Chi movements (such as standing on one leg) were not included in exercise program due to the safety of participants, so, we presume that this is a possible reason why no statistically significant changes were observed in one leg and tandem stance sections of BESS test. And finally, as Bell et al. (2011) stated, BESS may not be valid when differences are more subtle, which is consistent with our study results as "Sigma Balance Pad" was capable to measure these subtle balance changes.

\section{CONCLUSIONS}

Obtained results showed that body sway was less and center of pressure displacement speed decreased after Tai Chi exercise program, which means balance improvement. Current findings of BESS test results prompt the need for further research, which could be based on the establishment of the reliability and validity of the BESS in subjects with osteoporosis.

\section{REFERENCES}

Bell, D. R., Guskiewicz, K. M., Clark, M. A., \& Padua, D. A. (2011). Systematic review of the Balance Error Scoring System. Sports Health, 3(3), 287-295.

Brech, G. C., de Foncesa, A. M., Bagnoli, V. R., Baracat, E. C., \& D'Andrea Greve, J. M. (2013). Anteroposterior displacement behavior of the center of pressure, without visual reference, in postmenopausal women with and without lumbar osteoporosis. Clinics (Sao Paulo), 68(10), 1293-1298.

Curtis, E. M., Moon, R. J., Dennison, E. M., Harvey, N. C., \& Cooper, C. (2015). Recent Advances in the Pathogenesis and Treatment of Osteoporosis. 
Clinical Medicine (London, England) 15 (Suppl. 6), 92-96.

Lelard, T., Doutrellot, P. L., David, P., \& Ahmaidi, S. (2010). Effects of a 12-week Tai Chi Chuan program versus a balance training program on postural control and walking ability in older people. Archives of Physical Medicine and Rehabilitation, 91(1), 9-14. doi: 10.1016. apmr.2009.09.014

Li, F. (2014). The effects of Tai Ji Quan training on limits of stability in older adults. Clinical Interventions of Aging, 9, 1261-1268. doi: 10.2147/CIA.S65823

Rutkauskienè, L., Piščalkienè, V., Gintilienè, M., Zachovajevienè, B., \& Kavaliauskienè, A. (2012). Vyresnio amžiaus asmenu pusiausvyros vertinimas naudojant „Sigma Balance Pad“. Sveikatos mokslai, 22(5), 52-56.

Smulders, E., van Lankveld, W., Laan, R., Duysens, J., $\&$ Weerdesteyn, V. (2011). Does osteoporosis predispose falls? A study on obstacle avoidance and balance confidence. BMC Musculoskeletal Disorders, 12(1). doi: 10.1186/1471-2474-12-1

Wayne, P. M., Gow, B. J., Costa, M. D., Peng, C. K., Lipsitz, L. A., Hausdorff, J. M., . . . Manor, B. (2014). Complexity-based measures inform effects of Tai Chi training on standing postural control: cross-sectional and randomized trial studies. Public Library of Science, 9(12), e114731. doi: 10.1371/journal.pone.0114731

Wayne, P. M., Kiel, D. P., Buring, J. E., Connors, E. M., Bonato, P., Yeh, G. Y., . . . Davis, R. B. (2012). Impact of Tai Chi exercise on multiple fracture-related risk factors in post-menopausal osteopenic women: A pilot pragmatic, randomized trial. BMC Complementary and Alternative Medicine, 12(7). doi: 10.1186/1472-6882-12-7

Zhou, Z., Chen, C., Zhang, J., Ji, X., Liu, L., Zhang, G., . . . Wang, P. (2014). Safety of denosumab in postmenopausal women with osteoporosis or low bone mineral density: A meta-analysis. International Journal of Clinical and Experimental Pathology, 7(5), 2113-2122. 